\title{
Infection mycosique d'un kyste synovial du poignet
}

\author{
Par P. DESTOMBES, F. MARIAT et J.-P. ROUSSELOT
}

Le rôle des champignons en pathologie inflammatoire s'étend semble-t-il peu à peu et se manifeste dans des conditions diverses que nous enseigne l'épidémiologie générale des mycoses.

1) Un champignon introduit dans un organisme sain entraîne rapidement des manifestations pathologiques primitives, en général locales et qui évoluent habituellement vers la guérison. Telles sont les pneumopathies américaines qui suivent l'inhalation de spores d'Histoplasmes, de Coccidioïdes et de Blastomyces. Si l'organisme est en état de moindre résistance, des lésions graves et disséminées peuvent apparaître. Ces champignons sont donc doués d'un pouvoir pathogène permanent.

2) Certaines mycoses profondes cosmopolites, souvent généralisées, apparaissent à titre de manifestations secondaires chez des malades atteints de processus malins (maladie de Hodgkin, leucoses), de diabète ou de cirrhose ou d'autres états encore mal définis qui favorisent l'implantation, la croissance et la diffusion d'un saprophyte exogène ou endogène devenu pathogène: Candidoses, Mucor-mycose, Cryptococcose sont parmi les plus connues de ces infections secondaires, certaines formes d'Aspergilloses pouvant se développer plus spécialement dans une dilatation bronchique ou dans une caverne tuberculeuse. Un traitement au long cours par antibiotiques, antimitotiques et surtout par corticoïdes est un procédé « de choix » pour que ces saprophytes extériorisent un pouvoir pathogène virtuel jusque-là.

3) Une blessure peut introduire dans l'organisme un champignon du sol ou des plantes qui va, dans ce nouvel habitat, manifester un redoutable pouvoir pathogène. Certains ont une dispersion mondiale tel Sporotrichum schenckii, d'autres ont un biotope précis et les mycoses qu'ils entraînent ont alors une distribution géographique stricte, tels sont la plupart des agents fongiques ou actinomycosiques des mycétomes.

4) Enfin, on peut avoir la surprise de constater in vivo le rôle pathogène d'un champignon jusque-là connu comme saprophyte. C'est le domaine, chaque jour plus étendu, des champignons pathogènes occasionnels, les «opportunistics fungi » des auteurs anglo-saxons. 
L'observation qui suit en est une illustration :

Un tailleur de haies, exerçant son art le long d'un chemin près de Aulnay-sur-Odon en Calvados, a malencontreusement été piqué au poignet par une des branches de l'aubépine qu'il venait de couper. Or, ce poignet était le siège depuis des années d'un kyste synovial fort bien toléré. Dans les jours qui suivirent, ce kyste devint douloureux et, les soins médicaux ayant échoué, le malade se confia, aux environs du quinzième jour, au chirurgien qui fit l'ablation en bloc de la lésion et la fixa immédiatement.

La pièce d'éxérèse (examen BE 2009) montre des lésions considérables siégeant sous l'hypoderme ; localement, elles gagnent la surface où existe une petite ulcération. En profondeur, une large réaction inflammatoire chronique forme une lame onduleuse épaisse de 1.000 à $1.500 \mu$ qui constitue la paroi d'un kyste dont la cavité renferme des cellules desquamées dans une nécrose localement riche en polynucléaires. La membrane inflammatoire correspond à un granulome à corps étranger modérément et irrégulièrement suppuré, peu nécrotique, riche en macrophages multinucléés. Dans ce granulome, s'observe un champignon dont les filaments pigmentés sont disséminés sur toute l'étendue de la réaction inflammatoire, mais qui y restent bien confinés ; ils sont absents dans le derme sus-jacent qui ne réagit que très discrètement à un tel voisinage.

Ces filaments, isolés les uns des autres, se rencontrent aussi bien dans les plages suppurées que dans les zones histiocytaires et même une partie d'un filament peut être incluse dans plusieurs plasmodes successifs, tandis qu'une autre est entourée de polynucléaires.

Les tubes, cloisonnés et irrégulièrement ramifiés, ont une paroi pigmentée en brun foncé, mais les extrémités des hyphes peuvent rester hyalines. Le calibre est habituellement régulier, certains segments, cependant, sont dilatés et vésiculés, d'où parfois un aspect moniliforme. Des chlamydospores, assez fréquentes, peuvent être intercalaires ou isolées; dans ce cas, elles sont souvent englobées dans les cellules géantes.

Il n'existe aucune forme levure, les morphologies arrondies correspondent à des tubes coupés transversalement.

Au total, l'examen histologique indique que le kyste du poignet a été secondairement infesté par un champignon à paroi pigmentée qui pourrait appartenir au groupe des Dématiées.

A défaut de culture, l'histologie peut-elle tenter de situer le parasite?

Les maladies dues à des champignons pigmentés sont parfois groupées sous le nom volontairement vague de «Chromomycoses » (W. St-Symmers). C'est là pour l'histologiste, sinon pour le mycologue, un cadre assez commode. Parmi elles, on distingue :

1) La chromoblastomycose fréquente sous les tropiques. Il s'agit habituellement d'une dermatite verruqueuse, extensive, due à diverses espèces de Phialophora ( $P$. pedrosoi, $P$. verrucosa) et à une espèce de Cladosporium, $C$. carrionii. Quelle que soit l'espèce en cause, le champignon dans les lésions se présente sous la forme de «cellules fumagoïdes» en général peu nombreuses isolées ou groupées en petits amas, ce sont des 
PLANCHE I
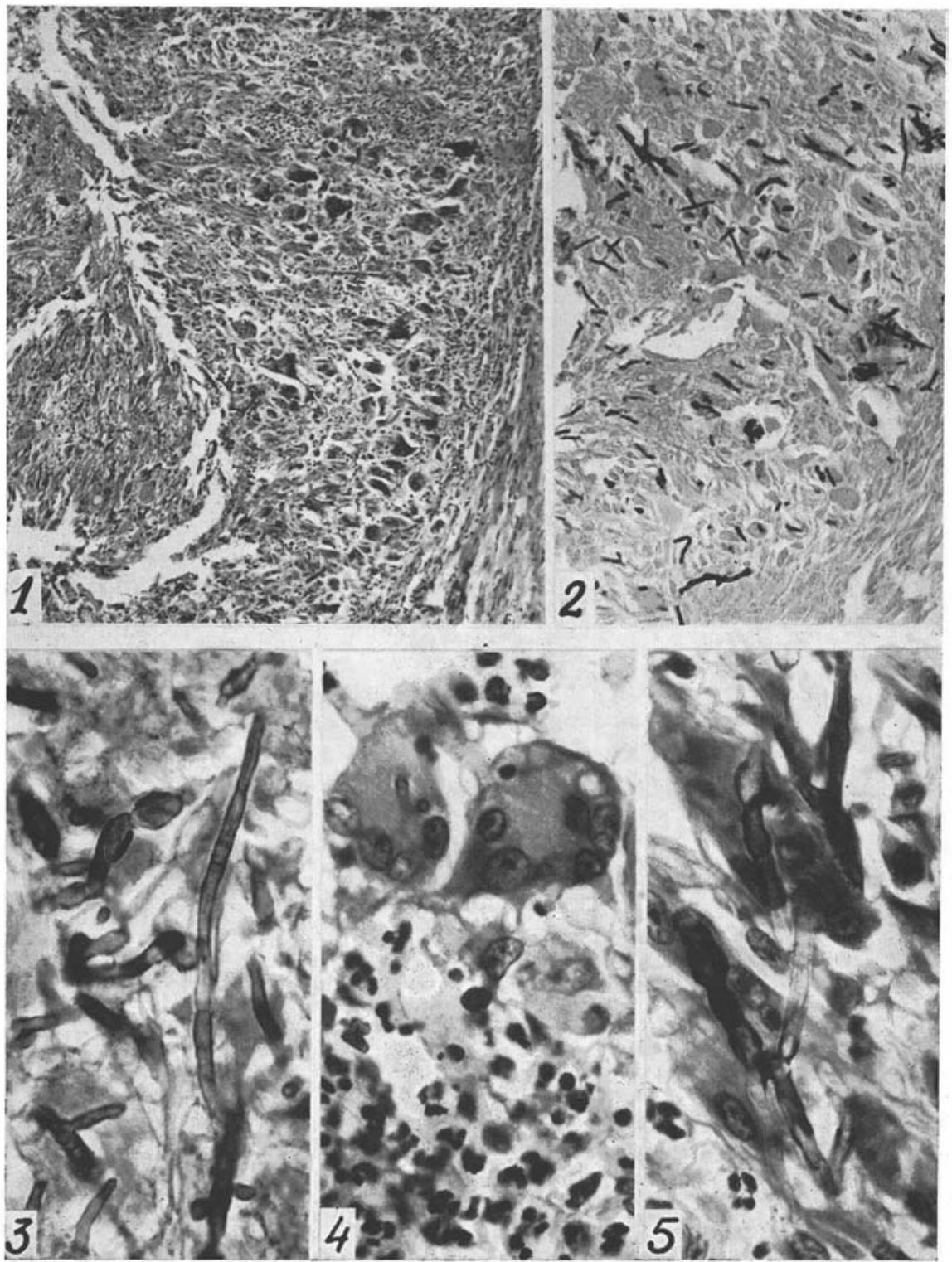

FIG. 1. - Arthrite mycosique. Epaisse lame inflammatoire granulomateuse riche en plasmodes à corps étranger et îlots suppurés. Quelques filaments à paroi pigmentée sont déjà visibles dès la coloration de routine. Coupe BE 2009 ; Hém. éosine-safran. $\times 72$.

Fig. 2. - L'imprégnation argentique-technique de Gomori-Grocottymet électivement en évidence les hyphes sans imprégner les fibres de réticuline.

Fig. 3 et 5. - Articles courts ou longs de filaments à paroi épaisse et pigmentée qui souvent devient brusquement hyaline. H.E.S. $\times 610$.

FIG. 4. - Ilots suppurés et zones macrophagiques $\times 610$. 
éléments pigmentés, arrondis, parfois divisés par une cloison transversale : il s'agit là de filaments et non de levures qui, elles, se reproduisent par bourgeonnement.

2) Dans un deuxième groupe de mycoses pigmentées, on retrouve d'autres espèces de Cladosporium, genre extrêmement répandu dans la nature. L'une $C$. wernekii reste épidermophytique et entraîne la Tinea nigra palmaire. Une autre espèce, $C$. trichoides ( $C$. bantianum) est souvent en cause dans les rares observations d'abcès cérébraux mycosiques. Une troisième enfin, est mal connue et son attribution générique a subi des vicissitudes nombreuses : elle a été successivement dénommée Sporotrichum, Phialophora et enfin Cladosporium gougerotii. Ce champignon a été rencontré dans des lésions sous cutanées et ostéo-articulaires et sa morphologie est très voisine de ce qu'on observe dans l'arthrite de notre malade; il est sans doute proche de Phialophora jeanselméi, celui-ci, agent de mycétomes à grain noir, peut aussi provoquer des mycoses souscutanées à filaments isolés. La question reste donc ouverte, car en l'absence de culture, l'histologie seule ne peut actuellement apporter un diagnostic étiologique.

Dans l'impossibilité d'isoler le champignon responsable à partir du matériel fixé, il nous restait l'espoir de l'isoler de la nature, à partir des végétaux mêmes qui étaient à l'origine de la lésion. Nous avons prélevé sur les arbustes (Crataegus oxyacantha) que taillait le patient lorsqu'il se blessa, des rameaux portant de nombreuses épines. La récolte a eu lieu le 19 décembre 1965 et, avec le matériel prélevé, les opérations suivantes ont été réalisées.

Quarante épines sont coupées en deux fragments : la pointe $(2 \mathrm{~mm})$ et le corps attenant à la pointe (environ $3 \mathrm{~mm}$ ). Les pointes lavées à plusieurs eaux stériles sont mises en culture sur deux lots de milieu glucosé de Sabouraud. L'un contient du chloramphénicol $(0,5 \mathrm{~g} / \mathrm{l})$, l'autre du chloramphénicol et de l'actidione (cycloheximide $0,5 \mathrm{~g} / \mathrm{l})$. Des tubes des mêmes milieux sont également ensemencés avec la première eau de lavage.

Les corps d'épines sont lavés une fois, macérés 24 heures dans de l'eau stérile, puis essorés et broyés au mortier dans $2 \mathrm{ml}$ d'eau physiologique. Le broyat est mis en culture sur les mêmes milieux que ci-dessus.

Dans tous les tubes (température du laboratoire), apparaissent en une semaine de nombreuses colonies fongiques. Sur les milieux sans actidione des espèces très diverses se développent: Aspergillus, Fusarium, Cladosporium, Phycomycètes, etc... et rendent toute analyse impossible. En revanche, en présence d'actidione l'observation est intéressante : douze des vingt pointes d'épines mises en culture donnent des colonies de champignons noirs à développement lent; une pointe donne une colonie d'Aspergillus. La première eau de lavage ne permet aucune culture. Le broyat des corps d'épines donne 24 colonies fongiques dont 21 de champignons noirs comparables à ceux obtenus à partir des pointes.

Afin d'éliminer les contaminants provenant de la surface des épines, de nouveaux fragments sont mis en culture après lavage à l'eau savonneuse suivis ou non de bains d'alcool à $96^{\circ}$. Des cultures des mêmes champignons noirs sont obtenues à partir des 


\section{PLANCHE II}
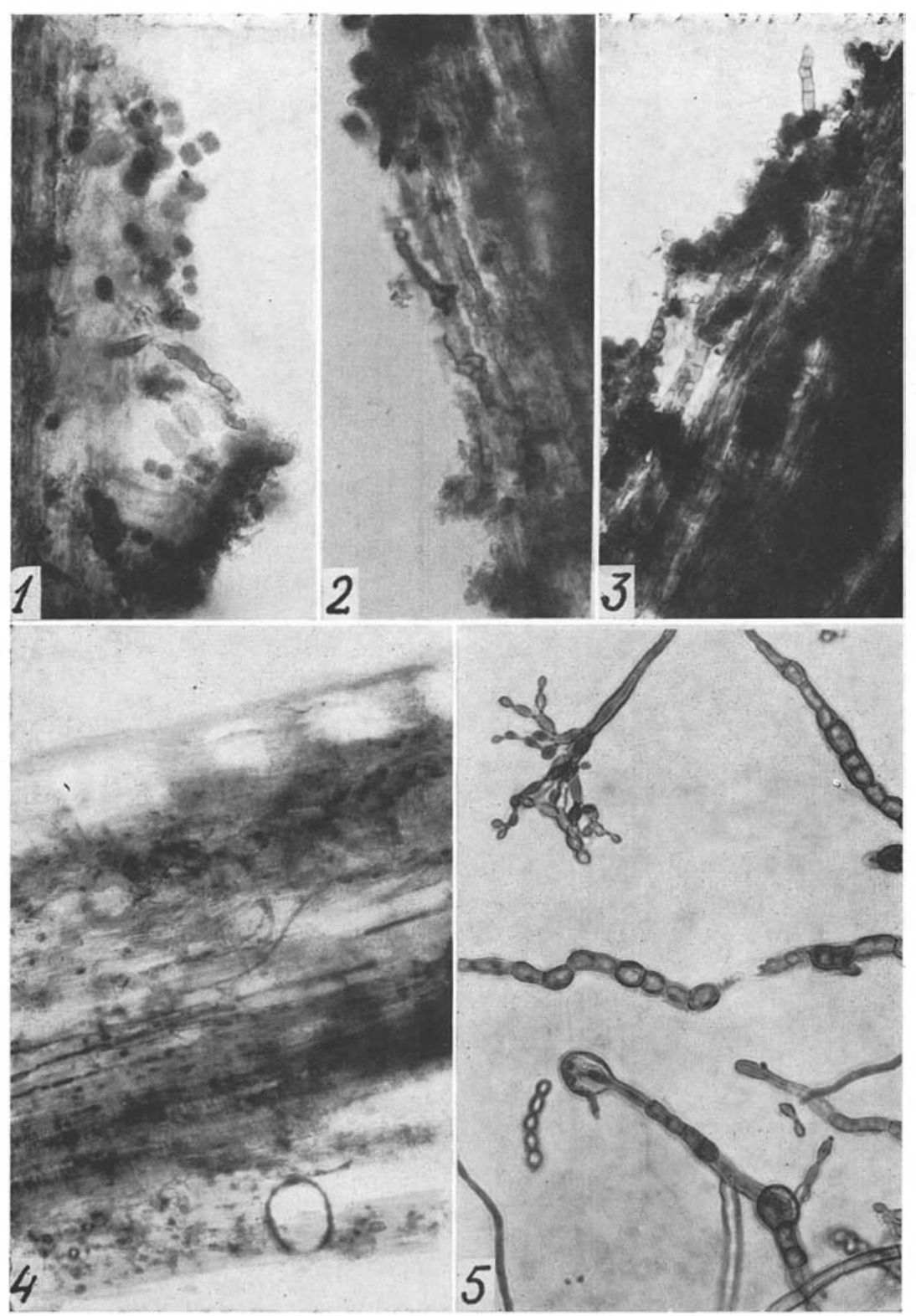

FIG. 1, 2, 3, 4. - Coupes d'épines avec filaments fongiques, à articles généralement courts et à parois brunes. On peut également voir (fig. 1) des algues unicellulaires. Ces dernières ne s'observent qu'en surface alors que les filaments pénètrent en profondeur.

Fig. 5. - Culture sur lame: filaments et appareil sporifére d'une des souches le plus fréquemment isolée (Cladosporium sp.).

Noter la ressemblance des morphologies fongiques représentées dans les diverses figures. 
épines lavées à l'eau de savon, mais le traitement à l'alcool empêche tout développement.

Les diverses colonies de champignons noirs ont été isolées et étudiées. La plupart des souches appartiennent à un Cladosporium à courtes chaînes de spores (planche II, fig. 5), dont les colonies sont gris-anthracite, duveteux à revers noir intense. Toutes les autres souches sont également d'un même groupe : colonies noir mat, d'abord lisses puis, avec l'âge, devenant duveteuses par secteurs ; l'examen microscopique montre des spores souvent bourgeonnantes, naissant du mycélium suivant le type Pullularia. Il pourrait s'agir d'Hormiscium. sp.

Aucun des champignons isolés ne se développe à $37^{\circ}$ et même cette température leur est fatale. Sur les nombreux tubes ensemencés et portés à $37^{\circ}$ pendant onze jours, quelques-uns seulement donnent une culture après incubation ultérieure à la température du laboratoire.

Certaines des souches sont inoculées dans les articulations des membres inférieurs de lapins (J.-C. Guillon). Dans un cas, on a retrouvé le champignon, localisé dans un tissu conjonctivo-adipeux présentant des plages inflammatoires histiocytaires plus ou moins plasmodiales.

Les filaments sont nombreux, mais les rétrocultures restent négatives.

En conclusion, ces observations permettraient de supposer que le champignon noir présent dans la lésion décrite était l'une des dématiées rencontrées avec une grande fréquence dans les épines de l'arbuste contaminateur. Le fait que les champignons isolés ne se développent pas à $37^{\circ}$ et aussi les essais infructueux d'inoculation expérimentale ne sont pas en faveur de cette hypothèse, mais ne permettent cependant pas non plus de l'éliminer en toute certitude. Il est en effet permis de penser qu'une des spores inoculées accidentellement présentait, par variation ou mutation, les propriétés physiologiques rendant possible un développement dans les conditions rencontrées dans le kyste. Nous nous proposons de poursuivre ces expériences avec du matériel prélevé à différentes périodes de l'année.

\section{Résumé}

Observation d'une arthrite mycosique du poignet secondaire à l'inoculation traumatique d'un kyste préexistant. Le champignon noir observé pourrait être l'une des dématiées isolées à partir des épines de l'arbuste contaminateur.

\section{(Institut Pasteur,}

Services d'Anatomie pathologique et de Mycologie

de Paris, 25, rue du Dr-Roux

et Faculté de Médecine de 67-Strasbourg

Institut d'Anatomie pathologique) 